\title{
Internet of Things based Low-Cost Real-Time Home Automation and Smart Security System
}

\author{
Kishore. $\mathbf{P}^{1}$, T. Veeramanikandasamy ${ }^{2}$, K. Sambath ${ }^{3}$ and S. Veerakumar ${ }^{4}$ \\ Department of ECS, Sri Krishna Arts and Science College, Coimbatore, TN, India ${ }^{1,2,3}$ \\ Department of EEE, Bannari Amman Institute of Technology, Sathyamangalam, TN, India ${ }^{4}$
}

\begin{abstract}
Many people today prefer smart devices which can be controlled remotely by the Internet rather than the manual control to improve the standard of living. The home appliances are expected to fully automatic and Internets of Things (IoT) is projected to provide dramatic improvements in smart home appliances. The internet technology is growing day by day and the internet connection is accessible everywhere in the world. The IoT is going to rule the world within a few years. This paper presents an Internet of Things based real-time home automation and security system using Arduino UNO and ESP8266 WiFi module which makes the system cost-effective and portable. It is used for controlling and monitoring home appliances (Fans, Lights, etc.) from anywhere in the globe using MQTT server. The home automation system includes an integrated temperature and humidity sensor module to control the air conditioning unit. The human motion detection is achieved by the PIR sensor for security purpose.
\end{abstract}

Keywords: Internets of Things (IoT); Home Automation; Arduino UNO; ESP8266 WiFi module; MQTT server; PIR Sensor.

\section{INTRODUCTION}

The Internet of Things (IoT) can be connecting various types of objects like smart phones, personal computer and tablets to the internet, which brings new fangled type of communication between things and things, and thinks and people [1].

Any man-made objects that can be assigned an IP address and it has the ability to transfer data successfully over a network, the interaction through a network is called IoT. The internet helps us to bring immediate solutions for many problems and able to connect from any of the remote places. The Internets of Things technology is used to come in with innovative idea and large development space for smart homes to improve the living standards of life [2, 3].

The growth of the Internet of Things will reform a number of sectors, like healthcare, automation, energy, transportation, etc. The cloud computing can be used in such case to implement the IoT infrastructure that augmented with sensors and actuators to monitor and control "things" from anywhere [4].

Remote home monitoring allows users to manage and control various aspects of home. These include motion detection, water leak detection, monitoring temperature against burglary and fire, and controls for lights, locks, fans and more from Laptop or Tablet or Smartphone [5].

\section{PROPOSED SYSTEM ARCHITECTURE}

The proposed home automation and security system consists of three modules: the hardware interface module, webserver and smart device. The hardware interface module includes Arduino UNO, ESP8266 WiFi module and relays which receive switching functionalities to control the home appliances connected to the system like Lights, Fans, Airconditioners and other appliances.

Once the system connected with WiFi home server the IP address is assigned to the ESP8266 by logging into WiFi modem. The IP address is typed in a web browser which will open the web page for home automation system. The communication between Arduino UNO and a webpage is handled by the web server. The Fig. 1 shows the architecture of proposed system.

The Adafruit IO cloud dashboard is created and a new gauge widget is linked with temperature feed and humidity feed. The WiFi module sends the data to Adafruit IO form Arduino UNO which will allow the user to monitor and control this home automation system from anywhere in the globe. Thus the home automation system allows different users to $\log$ on and access the webpage to control the appliances through a smart phone. 


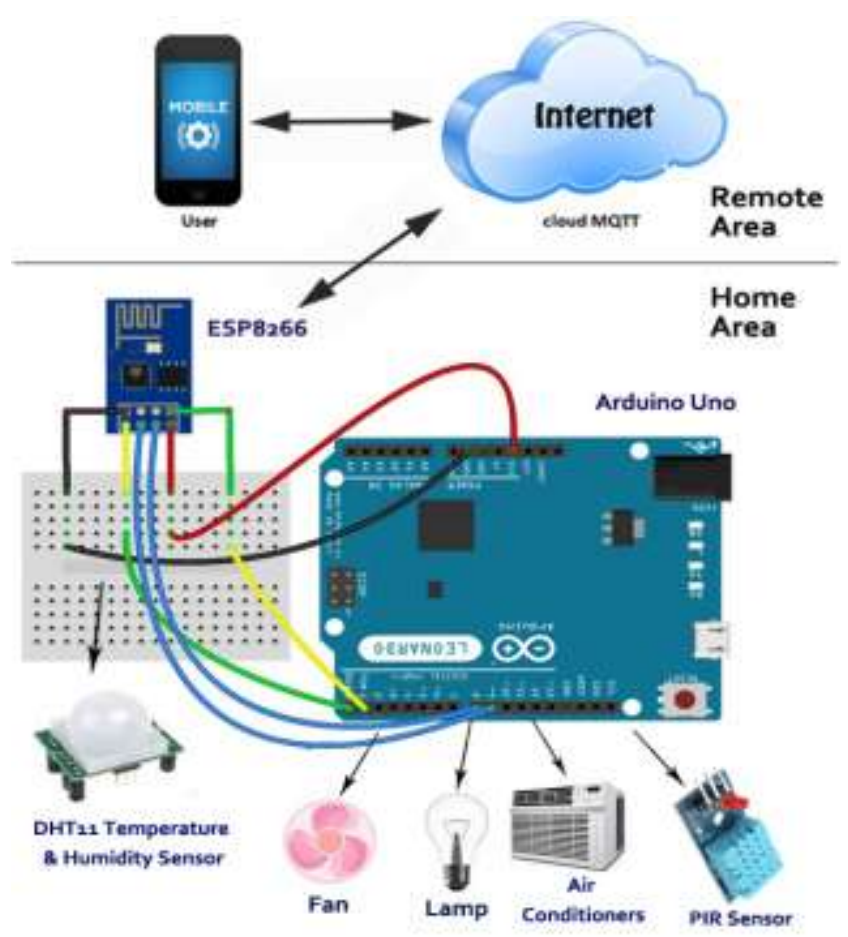

Fig.1: Proposed System Architecture

\section{SYSTEM IMPLEMENTATION}

The Fig. 2 shows the block diagram of IoT based a home automation system which consists of Arduino atmega328 open source hardware and ESP8266 WiFi module. It is interfaced with DHT11 temperature and humidity sensor, PIR human motion detection sensor and relays. The power supply provides 12V/1A DC current to Arduino UNO board, thus the board regulates the $12 \mathrm{~V}$ DC into $5 \mathrm{~V}$ DC and further into $3.3 \mathrm{~V} \mathrm{DC}$. From the Arduino UNO board the power is supplied to all I/O modules. The Arduino UNO is connected to DHT11 sensor and the sensor readings are continuously monitored by using serial protocol. The loads are connected to Arduino UNO through four channel relay board and ULN2003 driver board. The PIR human motion detection sensor connected to the WiFi module and the data are passed on to ESP8266 via serial UART. The ESP8266 is a transceiver which is connected to the Arduino UNO with 3.3V DC power, Tx transmitter and Rx receiver pins. The data are uploaded to the adafruits cloud service through WiFi module and which can control the device through the website called io.adafruit.com. The data are stored on the web server and monitor in the web page by creating virtual keys to each and every switches and sensors. The web page is able to control such loads by the Arduino UNO signals from the cloud service and monitoring the sensor value such as temperature, humidity and motion on the same web page. The end user web dashboard for home automation system is developed by using io.adafruit (cloud service) or blynk (android application).

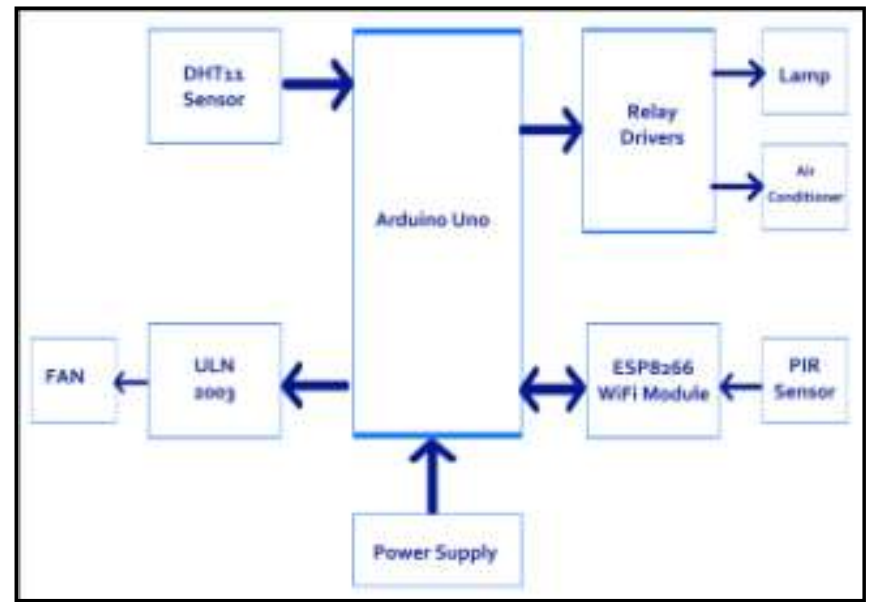

Fig. 2: Block diagram of IoT Based Real-Time Home Automation and Security System 


\section{A. ARDUINO UNO R3}

The Arduino Uno hardware is based on the ATmega328 with a flash memory of 32KB. It has 14 digital input/output pins out them 6 has PWM support, 6 analog inputs, a USB connection, a power jack, a $16 \mathrm{MHz}$ crystal oscillator and a reset button. It is operating with $5 \mathrm{~V} \mathrm{DC}$. The DC current per $\mathrm{I} / \mathrm{O}$ pins is $40 \mathrm{~mA}$ for $5 \mathrm{~V}$ and the DC current per $\mathrm{I} / \mathrm{O}$ pin is $50 \mathrm{~mA}$ for $3.3 \mathrm{~V}$.

\section{B. ESP 8266 WiFi Module}

The ESP8266 module offers an absolute and self-contained WiFi networking solution. It allows either to host the application or to offload WiFi networking functions from any other application processor. ESP8266 is an impressive, low cost WiFi module suitable for adding WiFi functionality to an existing microcontroller project via UART serial connection. The module can be reprogrammed for standalone WiFi device $[6,7]$.

\section{Sensors}

The PIR sensor is a pyroelectric type sensor which is used for detecting living objects. A Fresnel lens is mounted on a compact PCB, and using limited components to form the PIR sensor module. The delay time and Lux are adjustable and customization is accepted. The DHT11 sensor senses the temperature and humidity quantities by using the digital signal acquisition technique. This sensor includes NTC type temperature measurement and a resistive humidity measurement. It ensures high reliability, excellent long-term stability, connects to a high performance microcontroller, and offering excellent quality, fast response, anti-interference ability and cost-effectiveness.

\section{CIRCUIT DIAGRAM}

The circuit consists of the following sections: the power supply section, Arduino UNO based monitoring and controlling section and wireless transceiver section. The power supply circuit is inbuilt with Arduino UNO board. The ESP8266 WiFi module requires 3.3V DC supply therefore an IC LM1117A is used to supply 3.3V from 5V DC of Arduino UNO board. The appliances are interfaced to the Arduino UNO using ULN2003 Driver and relays. It has an internal flywheel diode to reduce back EMF. The PIR human motion detection sensor and DHT11 temperature and humidity sensor are used in this system. The PIR is connected to ESP Wi-Fi module and DHT11 is connected to the Arduino UNO board. The WiFi module collects the signals from the user through cloud io.adafruit, those signals are pass-through Arduino and controls the home appliances. The sensors send input signals to WiFi module and uploaded to cloud services io.adafruit or blynk. The Fig. 3 shows the circuit diagram of the proposed system.

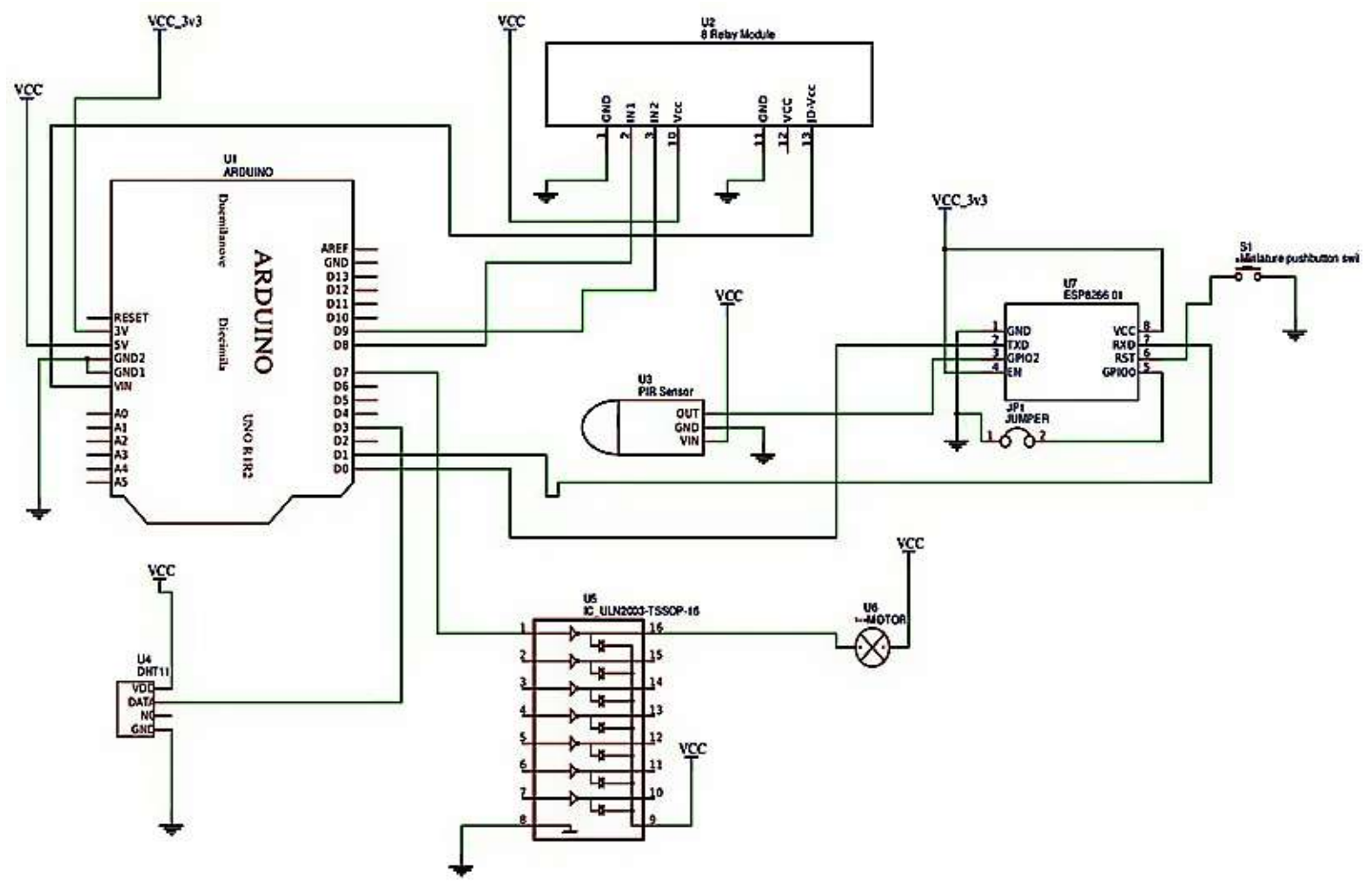

Fig. 3: The circuit diagram of proposed system 


\section{WEB APPLICATIONS}

The system can be accessed or interfaced through two ways one is by web page and another one by android application.

\section{A. IO ADAFRUIT Web Page}

Adafruit IO's MQ Telemetry Transport is a light weight message queuing and the transport protocol. MQTT can run on any kind of network, whether it will be TCP/IP or mesh network or Bluetooth.

Adafruit.io is open source MQTT server which is working on cellular and WiFi/ Ethernet connections, and TCP/IP connections. Firstly, the user has to sign in the ioadafruit.com for accessing the proposed system's web page and get the key code. The MQTT library and program for the Arduino is downloaded for interfacing WiFi module which is connected to the server and set a dashboard.

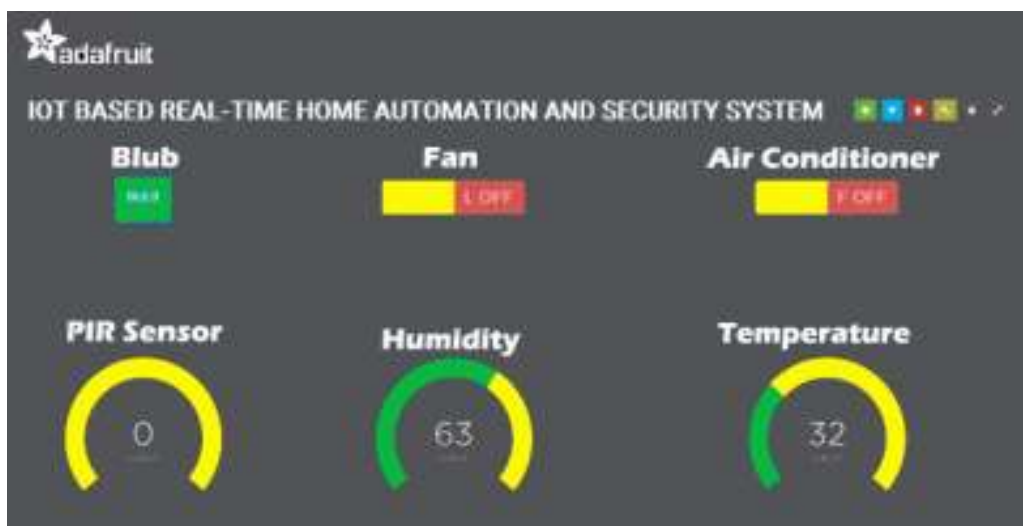

Fig. 4: Adafruit web page interface

To create the feeds, which belong to the things need to control and monitor. The Fig. 4 shows the ioadafruit web page interface of home automation system. The web page is also a convenient to manage and controls the things at home.

\section{B. ANDROID Application}

The android app is also can be used to control things very easily through the MQTT server. Here the appliances are controlled and monitor through a simple app called blynk. The Fig.5 shows the dashboard of blynk mobile app for IoT. It is an iOS and Android application. It is used to build a graphic interface for this system by simply dragging and dropping widgets on the digital dashboard. It controls Arduino, Raspberry Pi and other hardware over the Internet. Blynk app is getting connected to the internet and it is ready for the IoT application when the Arduino UNO is configured to the Internet over WiFi module.

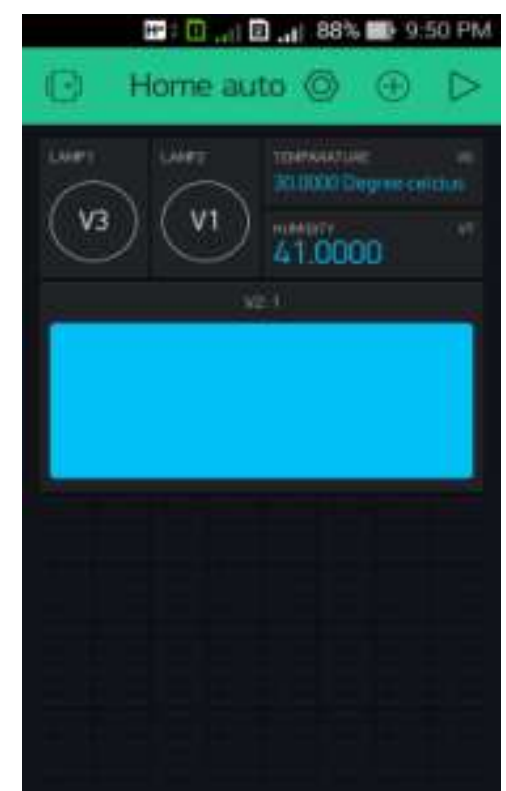

Fig. 5: blynk mobile app for IoT 


\section{CONCLUSION}

In this present work, a novel architecture for flexible home automation and security system using Internet of Things (IoT) has been implemented. The basic idea of "Internet of Things based low-cost real-time home automation and smart security system" is monitoring and controlling the home appliances by computer technology for the physically disabled people. The proposed system can be expanded to a variety of other options like capturing the photo of a living object moving around the house and storing on the cloud server. Further the image can be instantly sent to the user web page over the internet instead of storing in physical memory storage devices. This system can be incorporated with some additional changes and it is used in hospitals for disable people, in industries where human invasion is impossible and in environmental monitoring.

\section{REFERENCES}

[1] Pooja N.Pawar, Shruti Ramachandran, Nisha Singh, Varsha V.Wagh, A Home Automation System using Internet of Things, IJIRCCE, Vol. 4, Issue 4, April 2016.

[2] Somayya Madakam, R. Ramaswamy, Siddharth Tripathi, Internet of Things (IoT): A Literature Review, Journal of Computer and Communications, 2015, 3, 164-173.

[3] Chi-Sheng Shih, Jyun-Jhe Choum, Niels Reijers, Designing CPS/IoT applications for smart buildings and cities, IET Cyber-Physical Systems: Theory \& Applications, vol.1, Issue: 1, 122016.

[4] Federico Viani, Fabrizio Robol, Alessandro Polo, Wireless Architectures for Heterogeneous Sensing in Smart Home Applications: Concepts and Real Implementation, Proceedings of the IEEE, vol. 101, Issue: 11, Nov. 2013.

[5] Mamata Khatu, Neethu Kaimal, Pratik Jadhav, Syedali Adnan Rizvi, Implementation of Internet of Things for Home Automation, International Journal of Emerging Engineering Research and Technology, Vol. 3, Issue 2, February 2015.

[6] Dhakad Kunal, Dhake Tushar, Undegaonkar Pooja, Zope Vaibhav, Vinay Lodha, Smart Home Automation using IoT, International Journal of Advanced Research in Computer and Communication Engineering, Vol. 5, Issue 2, February 2016.

[7] Ahmed ElShafee, Karim Alaa Hamed, Design and Implementation of a WiFi Based Home Automation System, International Journal of Computer, Electrical, Automation, Control and Information Engineering Vol:6, No:8, 2012.

\section{BIOGRAPHIES}

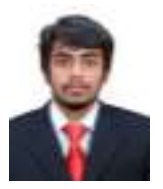

Kishore. $\mathbf{P}$ is currently pursuing his M.Sc. degree in Electronics and Communication Systems at Sri Krishna Arts and Science College, Coimbatore, received his B.Sc. Degree in Electronics at Govt. College, Chittur, Palakkad, Kerala in 2015.

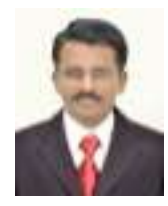

Dr. T. Veeramanikandasamy is an Assistant Professor at Sri Krishna Arts and Science College, Coimbatore, receive his Doctorate degree from Bharathiar University, Coimbatore in 2016. His involved researches are in the field of Nanomaterials Characterization and Embedded Systems.

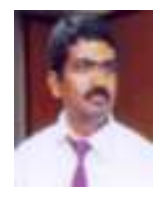

Dr. K. Sambath is presently working as Professor and Head in the Department of ECS, Sri Krishna Arts and Science College, Coimbatore, received his Ph.D Degree in Electronics from Bharathiar University, Coimbatore in 2012. His current research interests include Nanoscience and Embedded Systems.

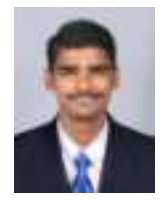

Dr. S. Veerakumar obtained his Doctorate degree from Anna University, Chennai in 2016. Currently, he is an Assistant Professor (Senior Grade) in Electrical and Electronics Engineering, Bannari Amman Institute of Technology, Sathyamangalam, Tamil Nadu, India. 